\title{
Transparência e a governança das águas
}

\author{
VANESSA LUCENA EMPINOTTI I, PEDRO ROBERTO JACOBI II \\ - ANA PAULA FRACALANZA III
}

\section{A introdução da transparência na gestão dos recursos hídricos}

$\mathrm{E}$ м 2008, a ONG Transparency International lançou o relatório Global Corruption Report, com foco nas práticas de corrupção ocorrendo no setor hídrico (Transparency International, 2008). Esse relatório trouxe, pela primeira vez, os conceitos de accountability e transparência para a gestão dos recursos hídricos como chave para combater a corrupção nesse contexto. Foi também nesse período que organizações multilaterais incluíram a transparência, accountability e o acesso à informação como peças-chave nas práticas de manejo e gestão dos recursos hídricos e como componentes da "boa governança" (UNDP, 1997; GWP, 2003; WWAP, 2003; OECD, 2015). Nesse contexto, a transparência é entendida como o acesso à informação a ser provida pelo Estado e qualquer outro ator envolvido nos processos de tomada de decisão que orientam o manejo dos recursos hídricos.

Tal estratégia reflete como a temática do controle sobre as instituições tem sido objeto de significativa difusão, destacando-se a ampliação dos argumentos em torno de governança participativa, governança democrática e "boa governança"; de controles democráticos, direito à informação e transparência; e em accountability (Lavalle; Vera, 2011). Dessa forma, o conceito de accountability adquiriu centralidade na teoria democrática contemporânea e dos Estados liberais, e explicita uma diferenciação entre o público e o privado. A premissa é que uma ordem política democrática se torna legítima mediante a responsabilização dos agentes públicos diante dos cidadãos (Filgueiras, 2011). Nesse sentido, conforme Przeworski e Cheibub (1999), esse conceito proporciona formas de gestão pública abertas à participação da sociedade.

Assim, a política da transparência e acesso à informação pode ser caracterizada como uma espécie de lugar-comum na política das sociedades liberais, que garantirá o funcionamento dos sistemas de gestão e a participação simétrica dos atores envolvidos uma vez que todos teriam o mesmo acesso às informações. Desse modo, a transparência torna-se um aspecto central de um processo democrático, porque coloca o tema da responsabilidade das instituições para que se reduzam as assimetrias de informação aos cidadãos. Isso coloca a necessidade, conforme Heald (2006), de que as instituições políticas informem e prestem 
conta aos cidadãos, e assumam a transparência como um valor instrumental para o exercício da accountability.

Entretanto, o que significa a existência ou não de transparência na gestão dos recursos hídricos? Como a qualidade dos dados disponíveis contribui na produção da realidade e nas ações propostas para solucionar os problemas? Neste artigo iremos apresentar e discutir como o conceito de gestão da transparência migra para a área de recursos hídricos e qual a sua contribuição dentro da perspectiva neoliberal de práticas públicas. Para fundamentar tal discussão, analisa-se como a transparência se transformou em um indicador de qualidade da prática da governança. Por fim, o artigo provoca os leitores a refletirem sobre como os resultados podem indicar não apenas mais uma prática de controle de governo, mas também como a falta de dados ou dados de má qualidade pode reorientar ações e contribuir para a desigualdade de acesso e distribuição da água nos países.

\section{Transparência no contexto da "boa governança" e a perspectiva neoliberal}

A importância do acesso à informação como parte das práticas para a promoção da participação na governança ambiental não é uma questão nova: já estava presente nas preocupações das lideranças mundiais quando da elaboração da Declaração do Rio de 1992, a qual definiu os Estados como responsáveis em facilitar e estimular a conscientização e a participação popular por meio da disponibilização de informações referentes às questões ambientais (United Nations, 1992). Isso se deve ao fato de o sistema de governança ambiental ser composto pelo elemento político, que consiste em balancear os vários interesses e realidades políticas; pelo fator credibilidade, instrumentos que apoiem as políticas, que faça que as pessoas acreditem nelas e pela dimensão ambiental. O processo de governança envolve múltiplas categorias de atores, instituições, inter-relações e temas, cada um dos quais suscetível a expressar arranjos específicos entre interesses em jogo e possibilidades de negociação, expressando aspectos de interesse de coletividades, com ênfase na prevalência do bem comum (Jacobi et al., 2015).

O conceito de governança não pode ser entendido, apenas, como uma construção ideológica, mas como exercício deliberado e contínuo de desenvolvimento de práticas cujo foco analítico está na noção de poder social que media as relações entre estado, sociedade civil e agentes econômicos e que podem ampliar os mecanismos de democracia participativa na medida em que as práticas oriundas das mediações poderão provocar e promover espaços antes inexistentes de participação social. Aqui se adota uma visão que identifica todos os esforços relacionados com a construção cultural para articular teorias, agendas, sujeitos e potencialidades, construção de alianças e cooperação, além de acumular energia para romper com as abordagens verticais e estanques das atividades humanas e transcender aquelas que se baseiam na supremacia do mercado (Jacobi, 2012). Isso configura um processo no qual se torna implícita a disseminação de alguns 
dos poderes centrais para instâncias do setor público mais próximas da escala local decisória, menos hierarquizadas, o que determina a inclusão de novos agentes, instituições e estruturas no processo decisório. Portanto, criam-se as condições para uma participação concertada entre vários representantes da sociedade civil na condução política e tomada decisória, em oposição à tradicional perspectiva top-down da administração centralizada (Jacobi et al., 2015).

Hoje, o acesso à informação traduz-se como transparência, um indicador de efetividade, integralidade e legitimidade das práticas de governança, e se transformou em um veículo para promover a diminuição da assimetria de conhecimento e consequentemente de poder no processo de tomada de decisão (Stalgren, 2006).

Tal discussão chegou ao contexto de governança da água por meio de relatórios de organizações como o Banco Mundial e a International Transparency que atestaram que a falta de transparência impactava diretamente a gestão das águas, tornando-se um fator preponderante nos esforços de melhoria e distribuição nas diferentes partes do mundo (Asís et al., 2009; Transparency International, 2008). Assim se entende que a governança da água deve ser fortalecida por meio da implantação de práticas participativas e da presença constante de transparência em todos os momentos do processo de tomada de decisão (Transparency International, 2008). Nesse sentido, a transparência foi apresentada como mais um princípio a ser considerado juntamente aos de participação e descentralização da gestão das águas, de maneira a garantir o acesso e a distribuição equitativa aos recursos hídricos (Asthana, 2008; Mitchell, 2011).

Em vários países, com destaque para os da América Latina, a institucionalização das práticas de transparência ocorreu por meio da criação de leis com o objetivo de garantir a disponibilização de informações pelo estado, assim como processos em que os cidadãos possam acessar informação. No Brasil, em 2009, foi regulamentada a disponibilização de informações referentes a gastos e finanças do Estado pela Lei Complementar n.131, assim como o acesso à informação pela Lei n.12.527 de 2011 (Empinotti et al., 2014).

Especificamente para o contexto dos recursos hídricos, a Lei Federal n.9.433/1997, que reformou a política nacional de gestão dos recursos hídricos, já estabelecia práticas de transparência como ações fundamentais para garantir o funcionamento da governança da água antes mesmo da promulgação das leis específicas sobre disponibilização e acesso à informação. A transparência é, portanto, elemento-chave nos vários momentos, tal como na disponibilização dos dados que embasam os instrumentos de gestão, na disponibilidade das etapas e dos documentos originados durante o processo decisório, tanto em colegiados de bacia como nos órgãos de Estado, e na aprovação e divulgação dos projetos e regras que orientam a gestão dos recursos hídricos no país.

Por fim, a transparência é vista como um componente essencial para promover equidade e justiça no processo decisório ambiental. Informação e sua 
divulgação configuram uma das principais estratégias de ativistas ao denunciar desastres ambientais provocados por práticas econômicas, como se caracterizam as ações do Greenpeace até hoje. Cabe ainda observar que na medida em que os cidadãos têm acesso à informação, podem se defender melhor em face dos diferentes tipos de riscos que podem afetá-los. Assim, os indivíduos impactados pelas ações de terceiros têm o direito de saber a qual risco eles estão expostos e o potencial de impacto sobre a sua saúde. Pode-se, portanto, afirmar que na maioria dos países em desenvolvimento os cidadãos poderiam ser empoderados para dar apoio ao governo na gestão compartilhada do meio ambiente se lhes fosse dado acesso à informação (Petkova; Maurer, 2005).

A transparência ocorre pelo acesso à informação que deve ser pública e disponível de forma fácil. Essa perspectiva ganha ainda mais evidência quando consideramos tal discussão dentro do contexto em que o surgimento de novas práticas de governança reflete os novos fluxos de informação e as suas diferentes maneiras de acesso e disseminação por meio de tecnologias como a internet, e mais recentemente as redes sociais $(\mathrm{Mol}, 2010)$. Nessas práticas, acesso à informação e conhecimento se tornaram chaves como estratégias para influenciar a tomada de decisão. Para a governança, acesso à informação pode reposicionar atores que terão maior impacto nos processos de negociação e discussão. Ao expor os problemas por meio de divulgação de dados, é possível aumentar o controle sobre os atores responsáveis pela execução de ações e os seus resultados, uma vez que muitos dos problemas ambientais contemporâneos são identificados e tratados a partir da divulgação de informações. Com isso, atores sociais com acesso à informação posicionam-se em vantagem com relação aos outros e se intensifica a discussão sobre práticas que levem à transparência (Mol, 2010; 2009).

Esse entendimento da transparência como uma ferramenta de empoderamento vai ao encontro da perspectiva liberal que valoriza as práticas de participação e descentralização. Ela reconhece a transparência como um meio para fortalecer e informar os setores marginalizados da sociedade e assim equalizar a assimetria de conhecimento e promover uma tomada de decisão mais justa e coerente de acordo com as demandas dos vários setores da sociedade. Assim, a transparência está diretamente relacionada com poder, uma vez que o seu objetivo é a democratização de informação pela disponibilização de informação ao prover um dos recursos mais poderosos nos dias de hoje, o acesso à informação e ao conhecimento (Gupta, 2010b; Mol, 2010).

Nesse contexto, a transparência se torna uma ferramenta para promoção e aumento da eficiência das práticas da boa governança, que leva a processos de tomada de decisão cada vez mais abertos para possibilitar o seu controle social. Entretanto, essa discussão não reconhece o sigilo como parte do processo de negociação. Uma vez que todas as informações são disponibilizadas, o uso desses dados e conhecimento não é controlado e eles podem ser utilizados de 
acordo com os interesses de cada ator, o que não necessariamente pode levar ao bem comum. Ao contrário, a disponibilização total de dados pode fortalecer aqueles atores com maior capacidade de análise e recurso de interpretação, assim recriando práticas de marginalização e assimetria de poder. Assim, é importante reconhecer os limites do acesso à informação e o papel do sigilo nos processos de tomada de decisão (Birchall, 2011). O acesso à informação sozinho não irá resolver a assimetria de poder entre atores sociais por si só, mas é um elemento-chave para construção das políticas públicas.

\section{O uso da transparência na gestão dos recursos hídricos hoje}

Além de entender a transparência como uma ferramenta de promoção da boa governança, o que observamos hoje é a sua adoção como um indicador de monitoramento e avaliação (OECD, 2015; Williams, 2015; De Stefano et al. (no prelo)). Nesse caso, a transparência é entendida como um instrumento dentro de um arcabouço de práticas com o intuito de avaliar o comprometimento dos atores sociais nos processos de tomada de decisão e ao mesmo tempo combater práticas de corrupção (Gupta, 2010a; Lavalle; Vera, 2011). Assim, a transparência surge como um dos instrumentos para avaliar a efetividade, integralidade e legitimidade das novas práticas de governança em que participação é um fator-chave nos processos democráticos, que contribuem para promover mais equidade, fortalecendo o nível de justiça social e bem estar dos cidadãos.

Uma das iniciativas que utilizam a transparência como indicador é o Índice de Transparência no Manejo da Água (Intrag), que consiste em uma avaliação do grau de transparência da gestão dos recursos hídricos por meio de informações disponíveis eletronicamente nas páginas oficiais dos órgãos gestores. Inicialmente aplicado ao contexto espanhol, foi depois ajustado e replicado no Brasil e em Portugal (relatórios, artigos e relatório 1 Brasil). A partir das questões que compreendem o questionário do Intrag, observou-se o tipo de informações disponíveis e como a sua disponibilização variou entre os diferentes contextos e anos analisados (De Stefano et al. (no prelo)).

Nesses estudos, a transparência tornou-se uma ferramenta de mensuração e avaliação das práticas, principalmente dos entes públicos e dos grupos em controle, relacionados ao uso e aplicação de recursos e investimentos. Entretanto, o monitoramento da transparência, por meio de indicadores traz uma série de limitações e até mesmo de riscos ao traduzir uma prática política em números. Isso porque os indicadores geralmente auxiliam a capturar detalhes mas não são capazes de identificar a relevância e o significado dos dados em um contexto mais amplo (Veenhoven, 2002). Ou seja, é possível identificar um número em uma escala de 0 a 10 quanto a informações disponibilizadas, mas esse número não considera as razões e os fatores que influenciam tal resultado. Por exemplo, um órgão gestor com baixo Intrag pode indicar um baixo número de dados disponíveis, entretanto as razões podem estar relacionadas à não disponibilização ou à inexistência de tais dados. 
Por fim, a disponibilização de informações pode ser manipulada ao oferecer análises tendenciosas dos números obtidos e assim desviar a atenção dos atores para questões irrelevantes (Gupta, 2008). Assim, o acesso à informação não é suficiente para garantir a participação informada durante o processo de tomada de decisão. É importante considerar o formato em que a informação é disponibilizada, sua linguagem, em que momento ela é disponibilizada e o quanto está atualizada (Mol, 2010).

Tal discussão nos leva a refletir sobre o significado da baixa disponibilidade de dados e da sua qualidade. Como a inexistência de dados ou a sua baixa qualidade orientam a produção de realidades que podem levar a práticas e soluções desencontradas? Na próxima seção discutiremos como a disponibilização de informações pode se tornar uma ferramenta de controle e, principalmente, como a disponibilização de informações de baixa qualidade fortalece o controle de atores sociais específicos e das soluções propostas para resolver problemas referentes ao acesso universal à água.

\section{Transparência e a baixa qualidade dos dados como ferramenta de controle}

Um dos principais argumentos para justificar a prática do acesso à informação e a accountability é a possibilidade de controlar como os gastos e investimentos estão ocorrendo. Dessa forma, seria possível não só identificar, mas também coibir desvios e práticas que possam beneficiar alguns grupos em detrimento de outros (Kolstad; Wigg, 2009). Tal entendimento reflete as práticas neoliberais de gestão que instrumentalizam o acesso à informação e ao mesmo tempo desconsideram os aspectos e consequências políticas de tais práticas.

Se analisarmos a institucionalização da disponibilização de informações a partir da perspectiva da governamentalidade (governmentality), poderemos entender que a transparência seria mais um instrumento de controle de governo, em que o cidadão seria o principal agente em monitorar e reforçar práticas do sistema que lhes governa. Isso faz sentido quando entendemos que as formas modernas de poder e regulação atingem seus efeitos não por forçar as pessoas em direção a objetivos definidos pelo Estado, mas por torná-los cúmplices do sistema ${ }^{1}$ (Agrawal, 2005; Foucault, 1994). Dessa forma, governamentalidade funciona por meio da construção de campos particulares de intervenção que legitimizam certos tipos de conhecimento, processos de identificação de problemas a serem resolvidos e suas práticas de solução ao transformá-las em "objetivas" e "naturais", enquanto servem para moldar e autorregular os indivíduos (Rose, 1999). Nesse caso, intervenções do Estado promovendo acesso à informação, assim como a participação são exemplos claros do racional contemporâneo liberal de governo que pretende operacionalizar as capacidades de auto governo dos governados na busca dos objetivos governamentais (Cruikshank, 1994). De acordo com esse raciocínio, o acesso à informação pode se tornar um instrumento disciplinar, organizando as relações entre o estado e os atores 
sociais, transformando-os em sujeitos de governo. Uma vez que os atores sociais participam, eles se tornam autorregulados como parte do sistema de governo.

Por outro lado, é chave reconhecer que a organização da realidade por meio de dados estatísticos cria e produz simplificações com a intenção de transformar populações e paisagem em um formato mais compreensível e calculável para então promover ações (Scott, 1998). Assim, a disponibilização de dados desatualizados e de baixa qualidade também pode ser considerada estratégia de controle. Tal entendimento nos possibilita uma maneira diferente de entender a transparência e os processos envolvidos. Uma vez que os dados apresentados não permitem a compreensão das condições reais da distribuição e qualidade da água manejada pelo sistema de gestão, as propostas de solução dos problemas são fragilizadas e a avaliação de sua efetividade é enfraquecida.

Por exemplo, se não temos claros quais os problemas existentes hoje em relação ao abastecimento de água de grandes cidades, não temos condições de identificar os fatores que levaram a tal situação. Assim, as soluções e ações propostas não irão resolver os problemas identificados e os programas de monitoramento não serão capazes de cumprir com suas funções. A falta de dados e de sua disponibilização contribuirá para o fortalecimento e controle de poucos atores sociais participantes do sistema de gestão, mas principalmente, contribuirá para a produção de uma realidade distorcida e que potencialmente orientará ações inadequadas que dificultam o acesso universal à água em qualidade e quantidade.

Pressupõe-se, contudo, que tal abertura e clareza de procedimentos esteja presente em todas as fases do processo: desde a publicitação de atos e atividades à disponibilização e divulgação de informação inteligível e acessível para o cidadão comum, à integração dos inputs da participação na decisão final, passando por um envolvimento ativo e equitativo dos vários stakeholders e demais interessados (Schmidt et al., 2015; De Stefano, 2012).

Dessa forma, a simplificação da realidade por meio de dados leva a intervenções que regulam o sistema de gestão em uma direção especifica (Scott, 1998). O entendimento dos processos a partir dos dados e conhecimento gerados torna-se peça-chave no controle e nas ações desenvolvidos na gestão dos recursos hídricos. Assim, a quantificação e análise da realidade é capaz de redefinir fronteiras entre política e objetividade que serão base para a realização de avaliações, priorização de problemas e alocação de recursos (Rose, 1999). Dessa forma, ao considerarmos a baixa qualidade dos dados podemos dizer que a realidade, já simplificada, se torna mais obtusa ainda, uma vez que redireciona e influencia o entendimento dos processos de forma tendenciosa (Truelove, 2015). A inconsistência e a falta de compreensão dos dados disponibilizados flexibilizam e moldam o conhecimento e as ações sobre a situação do abastecimento de água, como também as suas práticas de governança, produzindo assim a prática de “desmapear" e enfraquecer o diagnóstico dos problemas e suas soluções (Truelove, 2015). A falta de clareza, como também a disponibilização de dados 
problemáticos podem até confundir a avaliação da prática de transparência em um primeiro momento. A disponibilização poderia ser entendida como prova da ocorrência da transparência, entretanto as ações propostas a partir daí não garantirão o acesso à água de forma universal.

Podemos trazer tal discussão para a prática ao analisarmos a dinâmica de disponibilização e acesso à informação que ocorreu durante o caso da crise de abastecimento de água na Região Metropolitana de São Paulo, que resultou em danos ao provimento e acesso ao recurso, principalmente para o abastecimento populacional. Além da falta de um posicionamento claro pelo governo do estado de São Paulo sobre a adoção de medidas para o enfrentamento da situação de dificuldade de abastecimento, as informações transmitidas e recebidas pela população da Região Metropolitana de São Paulo, região essa que conta com cerca de 21 milhões de habitantes, se limitou ao volume dos reservatórios de água que abastecem a região. Ou seja, um exemplo claro de falta de transparência (Fracalanza; Freire, 2015).

De fato, a crise de abastecimento de água na Região Metropolitana de São Paulo somente foi anunciada pelo governo do estado de São Paulo em princípios de 2014, quando um dos sistemas de abastecimento de água para essa região (Sistema Cantareira) já estava com nível baixo de alguns de seus reservatórios. Entretanto, ela foi anunciada de forma lenta e pouco transparente. Pouco se soube e ainda pouco se sabe sobre como a população foi atingida pela crise.

Em pronunciamentos de representantes do governo de São Paulo o termo transparência era utilizado constantemente, uma vez que os dados do volume dos reservatórios dos sistemas que abastecem a região eram disponibilizados diariamente. Por outro lado, informações referentes ao mapa de distribuição da água na zona urbana não foram disponibilizadas por se tratarem, segundo o governo, de informações sigilosas. Por fim, quando foram realizados pedidos de informação via os dispositivos previstos pela Lei de Acesso à Informação, os dados eram disponibilizados em papel, em grande quantidade e com uma linguagem técnica de difícil entendimento.

Tais práticas exemplificam o que Jacobi, Cibim e Souza (2015) identificaram como práticas do governo do estado de São Paulo que prevaleceram durante o período da crise hídrica, em que a posição tomada é absolutamente contrária ao que se espera para se obter uma boa governança da água. Com um discurso absolutamente técnico e centralizador, o estado de São Paulo afastou qualquer integração com a população, podando o envolvimento da sociedade na discussão, tanto da causa da crise, como também das possíveis soluções para o enfrentamento do problema.

Ao negar o problema e não disponibilizar informações, o governo de São Paulo impôs uma realidade fabricada. Assim, a não disponibilização de informações, combinada à irrelevância dos dados apresentados, impossibilitou que a população atingida pela falta de água tivesse a informação necessária para lidar 
com essa situação. Dessa forma, o governo de São Paulo se tornou o principal tomador de decisão a partir de uma realidade embasada em informações disponibilizadas de baixa relevância e pelo controle total das ações.

\section{Conclusões: próximos passos no entendimento desses processos}

A transparência está fortemente associada ao "direito de saber". O surgimento de novas práticas de governança e a inclusão de atores sociais em novos espaços de negociação promovem a possibilidade de o acesso à informação não só se tornar um instrumento-chave, mas também assume uma importância como um fator de poder e influência sobre a tomada de decisão.

Toda boa comunicação interna e externa, particularmente quando espontânea, franca e rápida, resulta em um clima de confiança entre os participantes de um sistema de gestão com controle social. O maior desafio é de credibilidade e a necessidade de acordos que garantam a efetiva implementação dos princípios da política, para garantir os interesses e demandas das comunidades. O importante é o avanço do processo participativo e principalmente de aprendizagem. Nesse sentido a transparência e a preocupação com a prestação de contas são consideradas como condições necessárias para o sucesso de uma política pública no intuito de articular as ações de atores públicos independentes, visando objetivos comuns em um município, um estado ou em qualquer ambiente político ou de ação pública.

Ao mesmo tempo, a transparência é um componente essencial para promover equidade e justiça no processo decisório, com base num processo que informe adequadamente o público e estimule ao controle social. Esse pode ser entendido como a participação do cidadão na gestão pública: fiscalização, monitoramento e controle das ações da Administração Pública

Dessa forma, em um primeiro momento, a disponibilização de informações e seu acesso são reconhecidos como estratégias-chave para o fortalecimento e a eficiência da governança da água. Assim, atualmente, estudos sobre a prática de transparência na gestão dos recursos hídricos têm se concentrado em identificar a sua ocorrência.

Entretanto, é importante começarmos a explorar e entender as consequências da disponibilização das informações. Como a disponibilização de dados influencia a maneira como realizamos a gestão dos recursos hídricos? Qual o significado da falta de transparência? Quais os processos que são desencadeados a partir da disponibilização de dados e da sua qualidade? Verificar se o acesso à informação e os caminhos a ela existem é relevante e possibilita reconhecer práticas até então invisíveis e desconhecidas do processo de gestão (De Stefano et al. (no prelo)). Assim, é necessário que se avance no sentido de entender o que tais dados significam.

Por exemplo, os dados estão disponíveis, mas a sua qualidade é questionável. Como esse fato promove o desdobramento de práticas e a construção de realidades que fortalecem e direcionam maneiras específicas de tratar tais 
problemas? Como a disponibilidade de dados altera os processos de tomada de decisão? Qual a resposta dos órgãos gestores ao processo de monitoramento? Indicadores de avaliação são capazes de influenciar o tipo de informação a ser disponibilizada? São eles limitadores das práticas de transparência?

Por outro lado, também é importante entendermos o papel do sigilo na discussão de acesso à informação. A transparência total de informações de qualquer tipo pode ser apropriada por determinados atores e, em função da sua capacidade de análise e articulação, reconfigurar assimetrias de poder e beneficiar interesses específicos que, não necessariamente assegurem o bem comum.

Assim, novas perguntas são colocadas. Como o acesso à informação é mobilizado e apropriado pelos atores sociais? O que a transparência significa para os diferentes atores? Durante o período de seca em que o estado de São Paulo e principalmente a sua capital foram severamente impactados, o governo de São Paulo, por meio da sua Secretaria de Saneamento e Recursos Hídricos, entendeu que a disponibilização de dados referentes ao volume dos reservatórios que abastecem a região já se configurava como transparência. Como tais dados empoderam os atores sociais envolvidos? Quais dados são cruciais para a promoção desse empoderamento? Como tais dados são relevantes para a tomada de decisão? O volume representa o quanto de água está disponível nos reservatórios, mas não quanto está chegando nos estabelecimentos e residências e com que qualidade.

Estamos apenas no início da discussão sobre o papel do acesso à informação na governança da água. Por isso é importante reconhecermos as diferentes facetas e consequências de tais práticas. Como discutido várias vezes neste texto, o acesso à informação por si só não garantirá o acesso universal à água. É preciso entendermos os processos que ocorrem na disponibilização de tais dados, a sua qualidade e consequências.

Nota

I De acordo com Foucault (1991), governmentality é um conjunto formado por instituições, procedimentos, análises e reflexões, o cálculo e táticas que permitem o exercício do poder que tem sua população alvo como sua principal forma de conhecimento econômico político e como seu principal aparato de segurança. Governmentality está presente em todos os níveis da sociedade e impacta a vida de todas as pessoas.

\section{Referências}

AGRAWAL, A. Environmentality: technologies of government and the making of subjects. London: Duke University Press, 2005.

ASÍS, M. G. D.; O'LEARY, D.; LJUNG, P.; BUTTERWORTH, J. Improving transparency, integrity, and accountability in water supply and sanitation. Washington: World Bank, 2009. 
ASTHANA, A. Decentralization and corruption: Evidence from drinking water governance. Public Administration and Development, v.28, n.3, p.181-9, 2008.

BIRCHALL, C. Transparency, interrupted: secrets of the left. Theory, Culture and Society, v.28, n.7-8, p.60-84, 2011.

CRUIKSHANK, B. The will to empower: technologies of citizenship and the war on poverty. Socialist Review, v.23, p.29-55, 1994.

DE STEFANO, L. et al. Measuring information trasnparency in the water sector: what story do indicators tell? International Journal for Water Governance (no prelo).

DE STEFANO, L. et al. Public participation and transparency in water management. In: DE STEFANO, L.; LLAMAS, M. R. (Ed.) Water, agriculture and the environment in Spain can we square the circle? Leiden: CRC Press, 2012. p.217-25.

EMPINOTTI, V. L. et al. The role of stakeholders in water management in LAC. In: WILLAARTS, B.; GARRIDO, A.; LLAMAS, M. R. (Ed.) Water for food security and well-being in Latin America and the Caribbean. London: Routledge - Taylor and Francis Group, 2014. p.317-42.

FILGUEIRAS, F. Além da transparência: accountability e política da publicidade. Lua Nova, São Paulo, v.84, p.65-94, 2011.

FOUCAULT, M. Governmentality. In: BURCHELL, G.; GORDON, C.; MILLER, P. (Org.) The Foucault effect: studies in governmentality. Chicago: The University of Chicago Press, 1991. p.87-104.

. Power. New York: The New Press, 1994.

FRACALANZA, A. P.; FREIRE, T. M. Crise da água na região metropolitana de São Paulo: injustiça ambiental, privatização e mercantilização de um bem comum. GEOUSP, São Paulo, v.19, n.3, p.464-78, set./dez. 2015.

GUPTA, A. Transparency under scrutiny: Information disclosure in global environmental governance. Global Environmental Politics, v.8, n.2, p.1-7. 2008.

. Transparency to what end? Governing by disclosure through the biosafety clearing house. Environmental and Planning C: Government and Policy, v.28, p.12844, 2010a.

. Transparency in Global Environmental Governance: a coming of age? Global Environmental Politics, v.10, p.1-9, 2010b.

GWP - GLOBAL WATER PARTNERSHIP. Effective Water Governance: Learning from the Dialogues. GWP Secretariat. Stockholm, Sweden. 2003.

HEALD, D. Transparency as an instrumental value. In: HOOD, C.; HEALD, D. (Org.) Transparency: the key of better governance. Oxford: Oxford University Press, 2006.

JACOBI, P. R. Governança ambiental, participação social e educação para a sustentabilidade. In: PHILIPPI, A. et al. (Ed.) Gestão da natureza pública e sustentabilidade. Barueri: Manole, 2012. p.343-61.

JACOBI, P. R. et al. Governança ambiental. In: AMBRIZZI, T.; JACOBI. P. R.; DUTRA, L. Ciência das mudanças climáticas e sua interdisciplinaridade. São Paulo: Annablume, 2015. 
JACOBI, P. R.; CIBIM, J. C.; SOUZA, A. N. Crise de água na Região Metropolitana de São Paulo (2013-2015). GEOUSP, São Paulo, v.19, n.3, p.422-44, set./dez. 2015.

KOLSTAD, I.; WIIG, A. Is transparency the key to reducing corruption in resource-rich countries? World Development, v.37, n.3, p.521-32, 2009.

LAVALLE, A.; VERA, E. I. A trama da crítica democrática: da participação à representação e à Accountability. Lua Nova, São Paulo, v.84, p.353-64, 2011.

MITCHELL, R. B. Transparency for governance: The mechanisms and effectiveness of disclosure-based and education-based transparency policies. Ecological Economics, v.70, n.11, p.1882-90, Special Section - Earth System Governance: Accountability and Legitimacy. 2011.

MOL, A. Urban environmental governance innovations in China. Current Opinion in Environmental Sustainability, v.1, n.1, p.96-100. 2009.

MOL, A. P. J. Epilogue: the Future of Transparency: Power, Pitfalls and Promisses. Global Environmental Politics, v.10, p.132-43, 2010.

OECD. OECD Inventory. Water Governance Indicators and Measurement Frameworks. OECD Water Governance Initiative. 2015. Retrieved from http://www. oecd.org/gov/regional-policy/Inventory_Indicators.pdf

PETKOVA, E.; MAURER, C. Closing the gap, information, participation, and justice in decision making for the environment. Washington, DC: World Resources Institute, s. d. Disponível em: <http://pdf.wri.org/closing_gap_ch04.pdf>. Acesso em: 24 jun. 2005 .

PRZEWORSKI, A.; CHEIBUB, J. A. Democracy, elections, and accountability for economic outcomes. In: STOKES, S.; MANIN, B. (Org.) Democracy, accountability and representation. Cambridge: Cambridge University Press, 1999.

ROSE, N. Powers of freedom. Cambridge: Cambridge University Press, 1999.

SCHMIDT, L. et al. Índice de transparência na gestão da água em Portugal (Intrag). In: I CONLAB, 2015. Lisboa.

SCOTT, J. Seeing like a state: How certain schemes to improve the human conditionhave failed. London: Yale University Press. 1998.

STALGREN, P. Corruption in the water sector: Causes, consequences and potencial reform. In: SIWI, 2006. Stockholm.

TRANSPARENCY INTERNATIONAL. Global corruption report 2008: corruption in the water sector. Cambridge: Transparency International, 2008.

TRUELOVE, Y. Gray zones: water, power and everyday practice in Delhi, India. Inglaterra, 2015. Tese (Doutorado em Geografia) - Departamento de Geografia, University of Cambridge. 2015.

UNITED NATIONS. Report of the United Nations Conference on Environment and Development. Rio de Janeiro: United Nations. 1992.

UNDP. Governance for Sustainable Human Development, A UNDP Policy Document. New York: UNDP, 1997.

VEENHOVEN, R. Why social policy needs subjective indicators. Social Indicators Research, v.58, p.33-45, 2002. 
WILLIAMS, A. A global index of information transparency and accountability. Journal of Comparative Economics, v.43, n.3, p.804-24, 2015.

WWAP - WORLD WATER ASSESSMENT PROGRAMME. UN World Water Development Report 1. World Water Assessment Programme, Paris, 2003.

Agradecimentos à Fapesp (projeto Bluegrass, processo 2013/50537-0).

RESUMO - Este artigo apresenta e discute como o conceito de gestão da transparência é considerado na área de recursos hídricos e qual a sua contribuição dentro da perspectiva neoliberal de práticas públicas. Para fundamentar tal discussão, analisa-se como a transparência se transformou em um indicador de qualidade da prática da governança da água. Por fim, o artigo provoca os leitores a refletirem sobre como a disponibilização de informações pode indicar não apenas mais uma prática de controle de governo, mas também como a falta de dados ou dados de má qualidade podem reorientar ações e contribuir na desigualdade de acesso e distribuição da água nos países.

PALAVRAS-CHAVE: Transparência, Governança da água, Acesso à informação, Democracia. ABSTRACT - This article introduces and discusses how the concept of transparency is considered in the area of water resources, as well as the concept's contribution to public practices from a neoliberal perspective. To substantiate this discussion, we examine how transparency has become a quality indicator for water governance. Finally, the article urges readers to reflect not only on how the availability of information may become one more practice of control of government, but also on how the lack of data or low quality data may redirect actions and lead to inequality in country-wide access to, and distribution of water.

KEYWORDS: Transparency, Water governance, Access to information, Democracy.

Vanessa Lucena Empinotti é doutora, professora adjunta em Planejamento Territorial na Universidade Federal do ABC - UFABC. @ - v.empinotti@ufabc.edu.br

Pedro Roberto Jacobi é doutor, professor titular no Instituto de Energia e Ambiente/ PROCAM, Universidade de São Paulo. @ - prjacobi@usp.br

Ana Paula Fracalanza é doutora, professora adjunta na Escola de Artes, Ciências e Humanidades (EACH), Universidade de São Paulo. @ - fracalan@usp.br

Recebido em 31.8.2016 e aceito em 23.9.2016.

I Universidade Federal do ABC - UFABC, Santo André/São Paulo, Brasil.

II Instituto de Energia e Ambiente/PROCAM, Universidade de São Paulo, São Paulo/ São Paulo, Brasil.

III Escola de Artes, Ciências e Humanidades (EACH), Universidade de São Paulo, São Paulo/São Paulo, Brasil. 